\title{
A Reconfigurable Inkjet Printed Antenna on Paper Substrate for Wireless Applications
}

\author{
Hattan F. Abutarboush, IEEE Member and A. Shamim, IEEE Senior Member
}

\begin{abstract}
An inkjet printed reconfigurable multiband antenna printed on a low cost commercial photo paper substrate using two PIN diodes is proposed for the first time. A main radiator is designed to operate at $3.4 \mathrm{GHz}$ band whereas L-shaped and U-shaped radiating elements are designed to operate at 1.9 and 2.4 GHz, respectively. The antenna has a size of $30 \times 40 \times 0.44 \mathrm{~mm}^{3}$ and can cover most of the mobile and wireless bands between 1.5 to $4 \mathrm{GHz}$. A prototype of the proposed antenna is fabricated and measured. Measured and simulated results of $S_{11}$, radiation pattern, gain and efficiency are in good agreement. Measured results show that the antenna has a gain and efficiency of $2 \mathrm{dBi}$ and $50 \%$, respectively. A parametric study is proposed as a guidance to design the antenna for operating in other bands between $1.5 \mathrm{GHz}$ to $4 \mathrm{GHz}$.
\end{abstract}

Index Terms - Nanoparticles, Inkjet antenna, Reconfigurable antenna, Paper antenna, Multiband antenna, PIN diodes, IoT.

\section{INTRODUCTION}

With the advent of multi-standard and multi-function wireless devices, there is a need to investigate multi-band and reconfigurable antennas so that one design can work for numerous standards and applications. Due to this reason, reconfigurable antennas have received a lot of attention recently [1] - [10]. On the other hand, application such as Internet of Thing (IoT), require the antenna to be low cost (almost to disposable level), light-weight, mass production and conformal. All these requirements can be met by realizing multiband or reconfigurable antennas through inkjet printing technique on low cost substrates. Though, frequency-reconfigurable antennas have been studied before from different research groups [1] - [10], but most of the designs use rigid substrates such as Duroid and FR-4. Some of the designs do use organic materials as a substrate for the antenna such as Liquid Crystal Polymer (LCP) and PET [11] [13], however these materials do not qualify to be extremely low cost substrates. Paper material, which is now being investigated as an electronics substrate, can reduce the cost approximately ten times that of the polymers or plastic films mentioned above [14]. Some antenna designs have been designed on paper substrate recently [15] - [19] but none of these show reconfigurability feature.

In this work, a frequency reconfigurable antenna inkjet printed on a paper substrate is demonstrated. In addition to the main

Manuscript received April, 2018

Hattan F. Abutarboush is with the College of Engineering, Electrical Engineering Department, Taibah University, Madinah, Saudi Arabia.

A. Shamim is with the Computer, Electrical and Mathematical Sciences and Engineering (CEMSE) Division, King Abdullah University of Science and Technology (KAUST), Thuwal 23955-6900, Saudi Arabia.

Email: Hattan.Abutarboush@taibahu.edu.sa

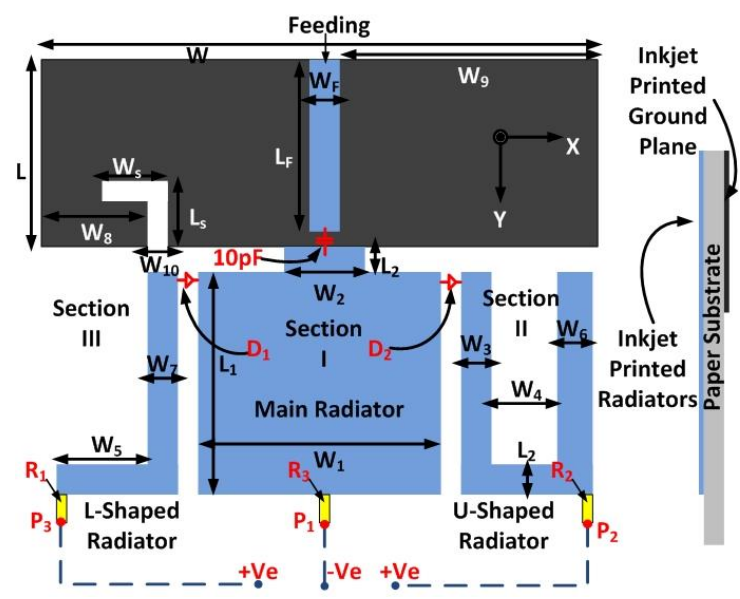

Fig. 1: Layout of the proposed antenna.

radiator, we used different shaped radiators to create two additional bands. The antenna is optimized to operate within the wireless and mobile bands from 1.5 to $4 \mathrm{GHz}$. With the help of PIN diodes, the proposed antenna can switch to various frequency bands of interest. Due to low cost substrate, and inkjet printing technology, this paper antenna can be used for a reasonable amount of time and can then be disposed off, making it highly suitable for IoT applications.

\section{ANTENNA Design}

\section{A. Antenna Structure}

The structure of the proposed antenna is shown in Fig.1. The microstrip feed line is designed to match 50-ohm impedance. The proposed antenna in Fig.1 consists of a main radiator, L-shaped radiator, a U-shaped radiator and a ground plane on the other side. The radiator is separated into three sections, section one is called the main radiator which is in a rectangular shape. Section II is called U-shape radiator and section III is called L-shape radiator. These shapes are designed to minimize the overall size of the antenna and to have multiple-band response. As a result, each section generates a single band. Then, these three sections are connected and disconnected using only two PIN diodes switches. These sections are fed by microstrip-line. To improve the matching of the antenna, a matching stub, as shown in Fig. 1, has been used as a transition from the feeding microstrip to the rectangular shape element. The antenna is designed on a resin-coated photo paper substrate with a thickness of $0.44 \mathrm{~mm}$, a dielectric constant of 3.2 and a loss tangent of 0.05 . The properties of the photo paper substrates and the ink were characterised earlier in [20]. The photo paper (from Kodak) has been a favourite choice for inkjet printed antennas. The antenna is designed using the high-frequency simulation software (HFSS). The optimized dimensions are listed in Table I. 
TABLE I: KEY DIMENSIONS OF PROPOSED ANTENNA IN MM

\begin{tabular}{|c|c|c|c|c|c|c|c|c|}
\hline $\mathrm{L}^{4}$ & $\mathrm{~L}_{1}$ & $\mathrm{~L}_{2}$ & $\mathrm{~L}_{\mathrm{f}}$ & $\mathrm{L}_{\mathrm{S}}$ & $\mathrm{W}$ & $\mathrm{W}_{1}$ & $\mathrm{~W}_{2}$ & $\mathrm{~W}_{3}$ \\
\hline 19 & 19 & 3 & 17.5 & 5.5 & 40 & 15 & 3 & 2 \\
\hline $\mathrm{W}_{4}$ & $\mathrm{~W}_{5}$ & $\mathrm{~W}_{6}$ & $\mathrm{~W}_{7}$ & $\mathrm{~W}_{8}$ & $\mathrm{~W}_{9}$ & $\mathrm{~W}_{10}$ & $\mathrm{~W}_{\mathrm{f}}$ & $\mathrm{W}_{\mathrm{S}}$ \\
\hline 5 & 9 & 3 & 3 & 12 & 17.5 & 2 & 1 & 8 \\
\hline
\end{tabular}

B. DC Biasing Circuit

A pair of PIN diodes, denoted as $D_{1} \& D_{2}$ in Fig. 1, have been used as switches for configuring the antenna to operate in different frequency bands. The L-shaped and main radiating elements are connected to the cathode and anode, respectively, of $D_{1}$, while the main and the U-shaped radiating elements are connected to the cathode and anode, respectively, of $D_{2}$. The diodes can be turned "ON" and "OFF" using a DC bias voltage of $+0.7 \mathrm{~V}$ and $-0.7 \mathrm{~V}$, respectively. To control the diodes states in our design, three $\mathrm{DC}$ wires are connected to the radiator elements via three resistors $R_{1}, R_{2}$ and $R_{3}$ with a value of 2.3 $\mathrm{k} \Omega$, as shown in Fig. 1 . These resistors are serve as RF chokes to prevent the RF signals in the radiators from getting into the DC-biasing circuit. The diodes used are practical and low-cost diodes, SMP1320-079, selected from Skyworks Solutions Inc. According to the data sheet, the diode has a resistance and capacitance of $0.9-\Omega$ and $0.3-\mathrm{pF}$, respectively, in the ON state and $3 \mathrm{k}-\Omega$ and $0.3-\mathrm{pF}$ in the OFF state. A slit is cut at the end of the feed-line and a chip capacitor of $10-\mathrm{pF}$ is placed over it for coupling the AC signal from the feed-line to the radiator. In computer simulation, the two diodes are modelled using the Resistance, Inductance, and Capacitance (RLC) boundary sheet.

The locations on the radiating elements for connecting the DC wires are obtained by intensive computer simulation. We studied the current distribution of the radiator at the operating frequencies of $1.95 \mathrm{GHz}, 2.4 \mathrm{GHz}$, and $3.4 \mathrm{GHz}$. We found that, at these frequencies, very little current occurs at the locations used for connecting the DC wires in the proposed design, thus the DC wires will have little effect on the antenna performance.

\section{Simulated AND MEASUREd $\mathrm{S}_{11}$}

The frequency reconfigurability of the inkjet antenna is studied using the $S_{11}$ reflection coefficient. The antenna shown in Fig.1 and the dimensions listed in Table I are used to print the antenna on the photo paper using a Dimatix DMP-2831 materials printer. A silver nanoparticle based ink from Sigma-Aldrich has been used. The detailed description of the printing and the alignment process can be found in [15-16]. In our fabrication of the antenna, we first print the structure of the antenna using metal nanoparticles ink on a Kodak paper and then place it inside an oven for 2 hours to heat the metal nanoparticles beyond the melting temperature. The metal nanoparticles ink then form continuous metal tracks. The SMA, PIN diodes, resistors and capacitance are then mounted on the antenna using low-temperature solder pastes and epoxies [16]. The antenna proposed in this paper cannot be flexible but it can be curved because any attempt to bend the antenna after the sintering process cracks in the printed structure will be visible as discussed in details in [16]. If the antenna is to be designed for curved surface, the procedure in [16] should be followed. The two PIN diodes offer four possible switching states, i.e., ON-ON, OFF-OFF, OFF-ON and ON-OFF.
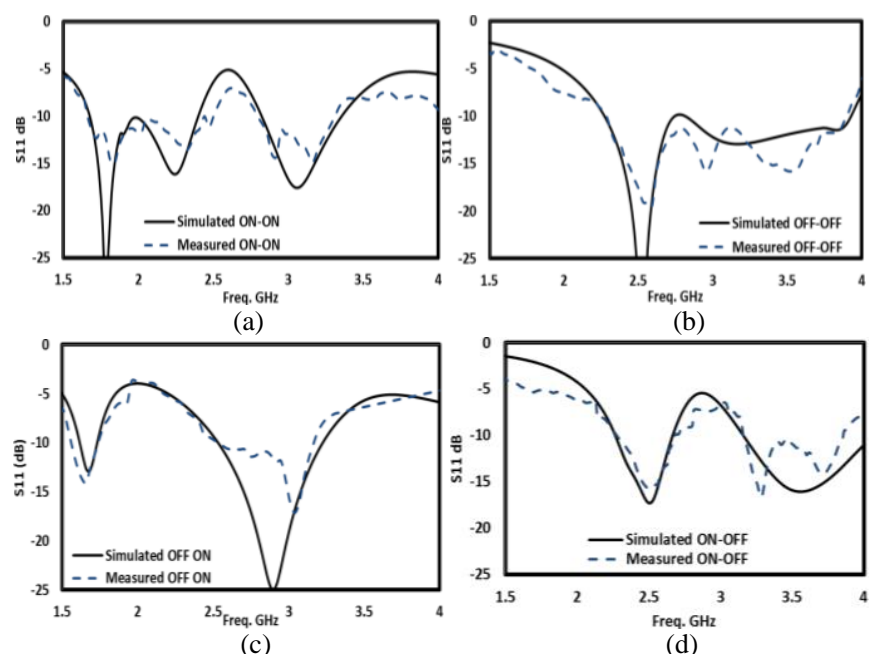

Fig. 2: Simulated and measured reflection coefficients $S_{11}$ of the antenna (a) ON_ON, (b) OFF_OFF, (c) OFF_ON, and (d) ON_OFF.

TABLE II: SimULATED AND MEASURED BANDWIDTH $\left(\mathrm{S}_{11}<-10 \mathrm{DB}\right)$

\begin{tabular}{|c|c|c|}
\hline & Simulated B.W $(\mathrm{MHz})$ & Measured B.W $(\mathrm{MHz})$ \\
\hline \multirow{2}{*}{ ON-ON } & $\mathbf{1 6 5 0 - 2 4 8 0}$ & $\mathbf{1 6 5 0}-\mathbf{2 5 0 0}$ \\
& $\mathbf{2 8 0 0 - 3 4 0 0}$ & $\mathbf{2 8 0 0}-3390$ \\
\hline OFF-OFF & $\mathbf{2 2 6 0}-3940$ & $\mathbf{2 2 6 0}-3900$ \\
\hline \multirow{2}{*}{ OFF-ON } & $\mathbf{1 6 1 0 - 1 7 9 0}$ & $\mathbf{1 5 6 0 - 1 7 9 0}$ \\
& $\mathbf{2 5 0 0 - 3 1 9 0}$ & $\mathbf{2 5 0 0}-\mathbf{3 1 7 0}$ \\
\hline \multirow{2}{*}{ ON-OFF } & $\mathbf{2 2 8 0 - 2 7 0 0}$ & $\mathbf{2 2 8 0}-\mathbf{2 7 2 0}$ \\
& $\mathbf{3 1 0 0}-\mathbf{4 0 0 0}$ & $\mathbf{3 1 0 0}-\mathbf{3 8 0 0}$ \\
\hline
\end{tabular}

By choosing the state of diodes, the U- and L- shape radiators will connect or disconnect to the main radiator, and operating frequencies will change. The simulated and measured results of the antenna are shown in Fig. 2(a)-(d). For easy comparison, the simulated and measured results for each state are plotted in separate figure. Figure 2(a) shows the simulated and measured reflection coefficients $S_{11}$ with the PIN diodes in the ON-ON state. When switching both PIN diodes ON hence both sections II and III are connected to Section I, the antenna can have dual-wideband as shown in Table II. The first and second bands were combined, due to the effect of the slot on the ground plane and formed a wideband with impedance bandwidth of $40.19 \%$ with respect to $\mathrm{S}_{11}<-10 \mathrm{~dB}$ as shown in Table II. The other band has an impedance bandwidth of $19.35 \%$. Therefore, the antenna in the ON-ON state can cover Personal Communication System (PCS), Digital communication Systems (DCS), Global System for Mobile Communications (GSM1900), (GSM 1800), Universal Mobile Telecommunications System (UMTS), LTE2300/2500 bands Wireless Local Area Networks $(2.4 \mathrm{GHz})$, Satellite Digital Multimedia Broadcasting (S-DMB), Worldwide Interoperability for Microwave Access (WiMAX) band II. In the OFF-OFF state, only section I is connected and functioning. In this case, the antenna has a wide band with respective bandwidth of $54.19 \%$ as shown in Fig. 2 (b) and Table II. When the antenna is in the OFF-OFF state, it can cover the following applications: WLAN, WiBro, WiMAX I and II, LTE2300/2500 bands and IEEE $802.11 \mathrm{y}$. For the OFF-ON state, only section II is connected to section I. The antenna in this case can have dual-band response centered at $1.9 \mathrm{GHz}$ and $2.9 \mathrm{GHz}$ with impedance bandwidth of $10.58 \%$ and $24.25 \%$ respectively 


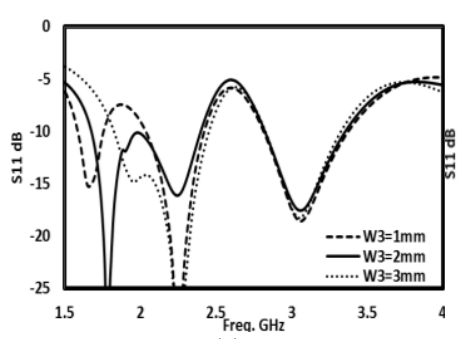

(a)

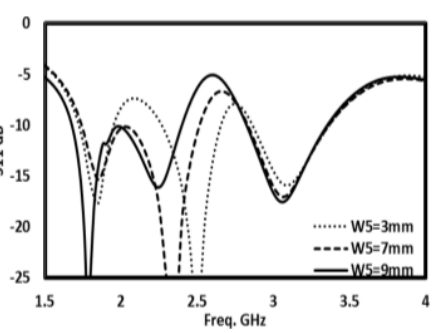

(b)

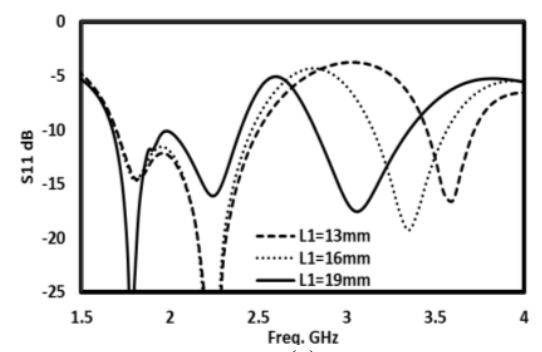

(c)

Fig. 3: Effect of some parameters on the $\mathrm{S}_{11}$ when the PIN diode is in the ON-ON state(a) $\mathrm{W}_{3}$, (b) $\mathrm{W}_{5}$ and (c) $\mathrm{L}_{1}$.

which can cover DCS, PCS, WLAN/Bluetooth, WiMAX band I, S-DBM and LTE2500. Figure 2 (d) shows the simulated and measured $S_{11}$ when the states of the PIN diodes are in the ON-OFF. In this case, section III is only connected to section I. It can be seen that dual-band can be obtained and centered at $2.4 \mathrm{GHz}$ and $3.4 \mathrm{GHz}$ with impedance bandwidth of $16.86 \%$ and $25.35 \%$ respectively. The antenna in the ON-OFF state can cover the WLAN, LTE2300, WiBro, S-DBM, and WiMAX I and II. Simulation results have shown that the stray capacitance and resistance have little effect on the antenna performance.

\section{PARAMETRIC ANALYSIS}

We investigated the impact of the related geometrical dimensions on the impedance matching condition of the antenna when it is in the ON-ON state only due to the limited space. Figure 3 (a) presents the simulated results of $S_{11}$ against the frequency of the proposed antenna with, $\mathrm{W}_{3}=1,2$ and 3 $\mathrm{mm}$. It can be seen that only the first resonant mode can be controlled and moves toward lower or higher frequency bands. However, when decreasing the size to $1 \mathrm{~mm}$, the wideband matching is lost. Another parameter that may affect the 1.9 $\mathrm{GHz}$ band is $\mathrm{L}_{2}$. For simplicity, we only showed the results of one parameter in this paper. Figure 3 (b) presents the simulated results of $S_{11}$ against the frequency of the proposed antenna when varying the dimension of $\mathrm{W}_{5}$ parameter. Here, with decreasing the size of the L-shape, the $2.4 \mathrm{GHz}$ band shifts toward a higher frequency bands because the effective electric length along the L-shape element is shortened when increases, whereas the other frequency bands are fixed and unaffected much. The wideband performance is seriously affected when reducing the size too much (less than $7 \mathrm{~mm}$ ) as shown in Fig. 3(b). The effect of changing the dimension of the length of the main radiator $\left(\mathrm{L}_{1}\right)$ is shown in Fig. 3(c). The simulated results of the proposed antenna with $\mathrm{L}_{1}=13,16$ and $19 \mathrm{~mm}$ are shown in Fig 3(c). We found that the resonant mode at $3 \mathrm{GHz}$ can be changed and controlled toward the higher frequency band, whereas the other resonant modes are almost fixed with slight affect in the bandwidth.

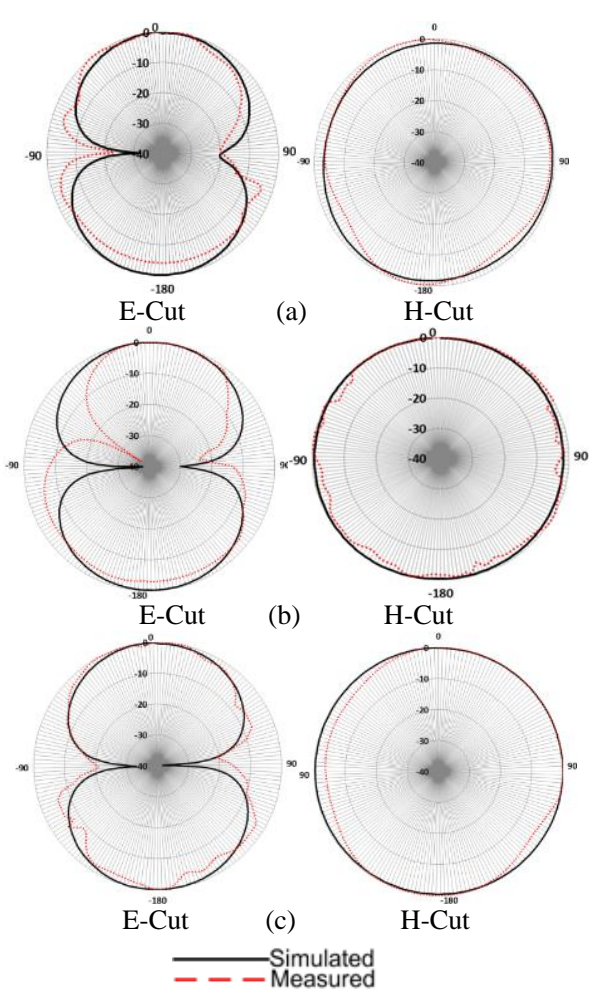

Fig. 4: Simulated and measured co- polrizations for E- and $\mathrm{H}$ - plane radiation patterns in the ON_ON state (a) 1.9 (b) 2.4 , and (b) $3.4 \mathrm{GHz}$.
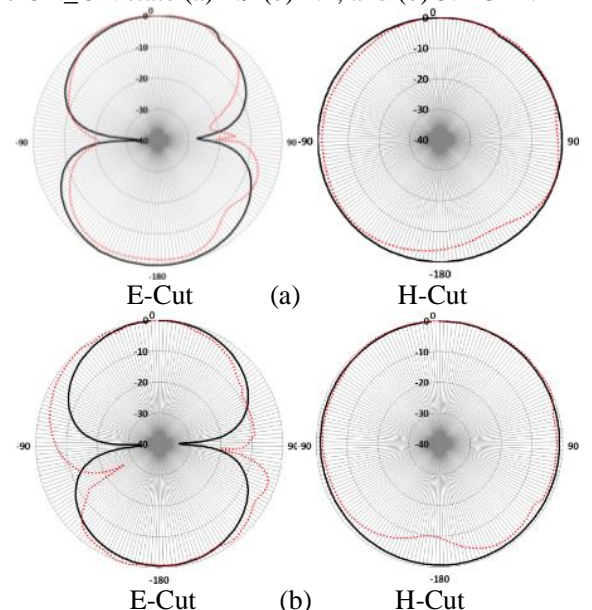

(a)

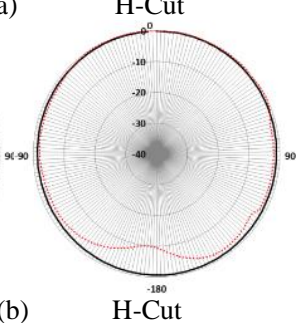

(b)

- Simulated

Fig. 5: Simulated and measured co- polarizations for $\mathrm{E}$ - and $\mathrm{H}$ - plane radiation patterns in the OFF_OFF state (a) 2.4 , and (b) $3.4 \mathrm{GHz}$.

\section{MEASURED RADIATION PATTERNS, GAIN AND RADIATION PATTERNS, GAIN AND EFFICIENCY}

The radiation pattern of the proposed antenna with different diodes states are measured using the equipment StarLab, manufactured by Satimo. Figs. 4 (a)-(c) and 5 (a)-(b) shows the normalized co-polarizations radiation patterns for $\mathrm{E}$ - and $\mathrm{H}$-.planes of antenna when the PIN diodes are in the ON-ON and OFF-OFF states, respectively. The simulated and measured results show a monopole-shape radiation pattern in the E-plane and an omnidirectional radiation pattern in the H-plane. The results presented in Figs. 4 and 5 show excellent agreement between the simulated and measured results. The radiation patterns at the other two states (OFF-ON) and (ON-OFF) have same performance and didn't change much. 

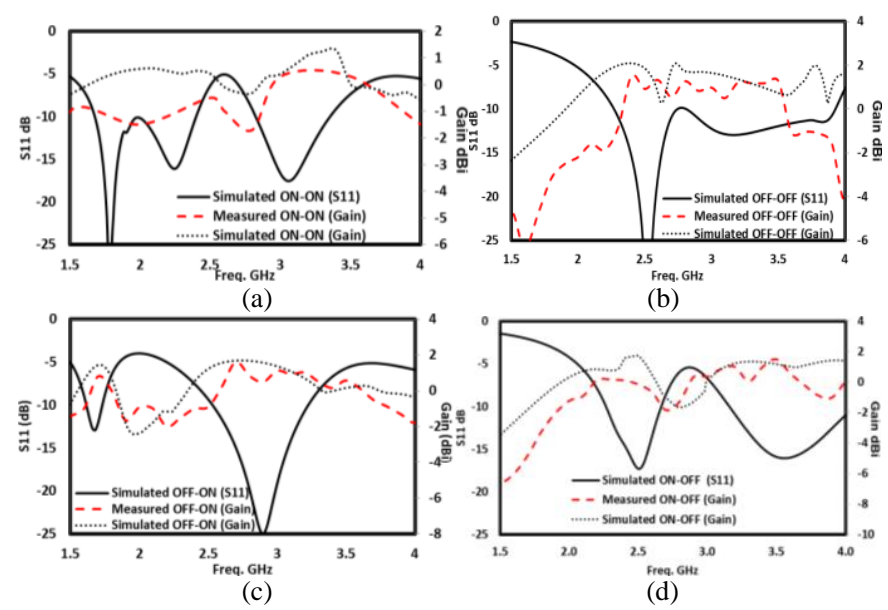

Figure 6: Simulated and measured gain versus frequency of the antenna (a) ON_ON, (b) OFF_OFF, (c) ON_OFF, and (d) OFF_ON.

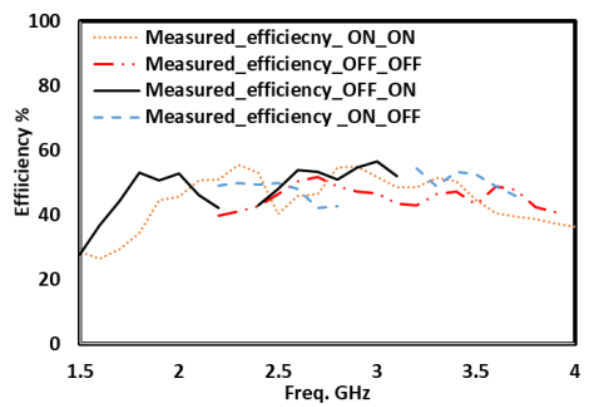

Fig.7 Measured efficiency of the antenna in the ON_ON, _OFF_OFF, OFF_ON, and ON_OFF states

To reduce the number of figures and for simplicity we did not include the results in the paper. Figures 6 (a)-(d) shows the simulated and measured gain of the antenna for the four states of the PIN diodes. Relatively good agreements between both curves can be observed within the operating frequencies. However, the largest discrepancy between simulated and measured gain occurs in Fig. 6(a) at the lower bands because both PIN diodes are in the ON state so the losses from the PIN diode and the paper material have resulted in larger discrepancies. The measured efficiency of the antenna is shown in Fig. 7, indicating that the efficiency is between $40 \%$ and 50\% which is decent efficiency for inkjet printed antenna on lossy substrate.

\section{CONCLUSION}

The design of an inkjet printed antenna with a compact size for a reconfigurable multiband and wideband wireless applications has been presented and experimentally demonstrated. L-shape and $\mathrm{U}$-shape radiators have been used to add additional bands and improve the bandwidth. Switching between the main radiator and the L- and U- shape radiators using two PIN diodes allowed the antenna to operate at different frequency bands. Simulation and measurement results have shown that the antenna has stable radiation pattern and moderate gain in all the operating frequency bands. The use of inkjet printing technology on a cheap paper substrate will reduce the overall cost of the antenna if considered to be produced for mass productions. The antenna can be easily optimised to operate at different frequency bands and more importantly the individual frequency band can be changed so that we can easily change them for other applications.

\section{REFERENCES}

[1] S. Yang, A. Kishk, and K. Lee, "Frequency reconfigurable U-slot microstrip patch antenna," IEEE Antenna and Wireless Propagation Letter, vol.7, pp. 127-129, 2008.

[2] D. Anagnostou, M. Khodier, J. Lyke, C. Christodoulou, "Fractal antenna with RF MEMS switches for multiple frequency applications," IEEE International Symposium Ante and Prop. Society, vol.2, pp.22-25, 2002.

[3] H. F. Abutarboush, R. Nilavalan, S. W. Cheung and K. M. Nasr, "Compact Printed Multiband Antenna with Independent Setting Suitable for Fixed and Reconfigurable Wireless Communication Systems," in IEEE Trans on Ante. and Prop., vol. 60, no. 8, pp. 3867-3874, Aug. 2012.

[4] Y. Tawk and C. G. Christodoulou, "A New Reconfigurable Antenna Design for Cognitive Radio," in IEEE Antennas and Wireless Propagation Letters, vol. 8, pp. 1378-1381, 2009.

[5] Lei Ge; Kwai-Man Luk, "A Band-Reconfigurable Antenna Based on Directed Dipole," IEEE Trans on Ante and Prop., vol.62, no.1, pp.64-71, 2014.

[6] D. Anagnostou, Z. Guizhen, M. Chryssomallis, J. Lyke, G. Ponchak, J. Papapolymerou, C. Christodoulou, "Design, fabrication, and measurements of an RF-MEMS-based self-similar reconfigurable antenna IEEE Trans on Ante and Prop, vol.54, no.2, pp.422-432, 2006.

[7] D. Anagnostou, A. Gheethan, "A Coplanar Reconfigurable Folded Slot Antenna without Bias Network for WLAN Applications," IEEE Antennas and Wireless Propagation Letters, vol.8, pp.1057-1060, 2009.

[8] H. F. Abutarboush, R. Nilavalan, K. Nasr, S. W. Cheung, T. Peter, H. Al-Raweshidy, and D. Budimir, "Reconfigurable tri-band H-shaped antenna with frequency selectivity feature for compact wireless communication systems," IET Microwave Antennas Propagation, vol. 5, no. 14, pp. 1675-1682, 2011.

[9] N. Behdad and K. Sarabandi, "Dual-band reconfigurable antenna with a very wide tunability range," IEEE Trans. Ante. Propag., vol.54, no.2, pp.409-416, 2006.

[10] H. F. Abutarboush, R. Nilavalan, S. W. Cheung, K. M. Nasr, T. Peter, D. Budimir, and $\mathrm{H}$. Al-Raweshidy, "A reconfigurable wideband and multiband antenna using dual-patch elements for compact wireless devices," IEEE Trans. Antennas Propagation, vol. 60, no. 1, pp. 36-43, Jan. 2012.

[11] N. Kingsley, D. Anagnostou, M. Tentzeris, J. Papapolymerou, "RF MEMS Sequentially Reconfigurable Sierpinski Antenna on a Flexible Organic Substrate With Novel DC-Biasing Technique," Journal of Microelectromechanical Systems, vol.16, no.5, pp.1185-1192, Oct. 2007.

[12] D. Anagnostou, "Flexible, wideband, dual-band and reconfigurable direct-write folded-slot antennas," URSI General Assembly and Scientific Symposium of Inter. Union of Radio Science, Istanbul, Turkey, Aug. 2011.

[13] S. M. Saeed, C. A. Balanis and C. R. Birtcher, "Inkjet-Printed Flexible Reconfigurable Antenna for Conformal WLAN/WiMAX Wireless Devices," in IEEE Ante and Wir. Prop. Let, vol. 15, pp. 1979-1982, 2016.

[14] Steckl, "Circuits on cellulose," IEEE Spect., vol.50, no.2, pp.48-61, Feb. 2013.

[15] H. F. Abutarboush and A. Shamim, "Paper-Based Inkjet-Printed Tri-Band U-Slot Monopole Antenna for Wireless Applications," in IEEE Antennas and Wireless Propagation Letters, vol. 11, pp. 1234-1237, 2012.

[16] H. F. Abutarboush, M. F. Farooqui and A. Shamim, "Inkjet-Printed Wideband Antenna on Resin-Coated Paper Substrate for Curved Wireless Devices," in IEEE Antennas and Wireless Propagation Letters, vol. 15, pp. 20-23, 2016.

[17] G. Shaker, S. Safavi-Naeini, N. Sangary, and M. Tentzeris, "Inkjet printing of ultrawideband (UWB) antennas on paper-based substrates," IEEE Antennas Wireless Propagation Letter, vol. 10, pp. 111-114, 2011.

[18] A. Rida, L. Yang, and M. Tentzeris, "Design and characterization of novel paper-based inkjet-printed UHF antennas for RFID and sensing applications," IEEE ISAP, pp. 2749-2752, 2007.

[19] E. Arnaud, A. Kanso, T. Monediere, D. Passerieux, M. Thevenot, E. Beaudrouet, C. Dossou-Yovo, and R. Noguera, "Inkjet printing of frequency selective surfaces on EBG antenna radome,". 6th EUCAP, pp. 2693-2696, 2012.

[20] B. S. Cook and A. Shamim, "Inkjet Printing of Novel Wideband and High Gain Antennas on Low-Cost Paper Substrate," in IEEE Trans on Ante and Prop, vol. 60, no. 9, pp. 4148-4156, 2012. 